\title{
Scheduling Algorithm Based on Logistics Random Graph Theory
}

\author{
Jing Li and Haiyun Peng \\ Institute of Computer Science and Technology, ZhouKou Normal University, \\ Zhoukou,Henan ,466001, China \\ zknulj@163.com
}

\begin{abstract}
Logistics from the user to pass character customization items, it has a certain degree of randomness and comprehensive, and the source node stream as nodes of the network, but how logistics resource scheduling can be more economical to transport goods, and because information technology, especially the development of things, dynamic and timely logistics scheduling becomes possible, for this problem, we integrated logistics dynamic scheduling features, applications random graph theory, based on a complex system of logistics scheduling scheme, simulation results show that the algorithm The advantages.
\end{abstract}

Keywords: Scheduling information; dynamic scheduling; random graph theory; information flow

\section{Introduction}

With the rapid development of the world economy and the progress of modern science and technology, the logistics industry in the national economy as an important service industries are growing rapidly worldwide, and has become the basis for development of industries and arteries of the national economy, has become a measure of the extent of its development one important indicator of a country's modernization and comprehensive national strength. Logistics industry known as the country's economic development, "accelerator", the evolution of industrial structure "lubricant" modern "the third profit source." According to China's WTO commitments, the logistics industry and the services sector is one of the earliest open industry. However, China's logistics industry is still in its infancy, there is a big gap with the developed countries, the most prominent problem is the high cost of logistics. 2010 China's total logistics cost of up to 7.453 trillion yuan, accounting for $16.8 \%$ GDP, while the United States and other developed countries, logistics costs account for about 10\% GDP, the proportion of moderately developed countries (such as South Korea, Singapore) is only about 13\%, which It shows that the overall level of China's logistics industry is seriously lagging behind. High logistics costs, restricted the development of the national economy, weakening the company's competitiveness in the market for China's logistics costs reduced by $1 \%$, representing an increase of more than 700 million economic benefit. Therefore, by improving logistics management, reduce logistics costs, can bring considerable economic benefits for business and society, to improve the operating efficiency of the national economy, improve international competitiveness. Supply chain efficiency is the impact made by a manufacturer of products in the market competitiveness. General supply chain around the core business is established, through the logistics, information flow and capital flow management and control, procurement of materials from suppliers began to be processed into intermediate products to the final finished product, and then to distribution channels, through network of retailers to sell finished products to consumers. This process, customers, retailers, distributors, manufacturers and suppliers formed a collaborative chain structure. Supply chain is a value-creating groups, materials suppliers through manufacturers to end users, that is, supply chain value creation process. 
Currently, the study of logistics problems still remain in the traditional distribution model, but with the rapid development of chain stores and e-commerce, logistics and distribution model has undergone tremendous changes, joint distribution, dynamic distribution, cross-regional large-scale distribution, etc. the new distribution of demand, the traditional distribution model has been difficult to support the needs of modern logistics and distribution. Especially the emergence of communication technologies and cloud computing technology, making large-scale cross-regional joint distribution as possible, the new distribution model will soon be born. Study on vehicle scheduling problem is still the main stay on a static vehicle scheduling problems, and the study of dynamic vehicle scheduling problem is still in its infancy. Operational research point of view, the dynamic and static problems are essentially different issues, the need to propose new solution strategies and solutions. Dynamic optimization of vehicle scheduling problem with time-related information, input information systems over time or change the appearance. When a new message, you need to quickly find a workable solution within an acceptable time frame, it also means go ahead with a pre-optimization method is computationally infeasible.

Postal delivery problems in the production and life, bus scheduling, power scheduling problems, plumbing problems, computer network topology design problem and so can be abstracted into a similar problem with the vehicle path combinatorial optimization problem. In this study, a dynamic vehicle scheduling problem for the study, from the perspective of systems engineering and analysis of cloud computing and social similarities logistics, distribution logistics made the cloud model, cloud distribution model to study the distribution of resources and customer optimal configuration requirements, the actual operation of logistics and distribution provide theoretical support. From the value point of view, the actual logistics and distribution companies in the production or distribution of customer demand for distribution by the team, rarely considering the cost savings, reduce waste of resources and improve the quality of service issues, or due to the complexity of the dynamic vehicle scheduling theoretical studies have been difficult to put into practice, leading to inefficient distribution of vehicles, the present study intends to vehicle scheduling problem for cloud distribution study, a mathematical model in line with the actual operation of operational optimization objectives and constraints, flexible design use intelligent vehicle dispatching system to provide support for logistics planning and vehicle scheduling optimization. Currently competition among enterprises has evolved into the competition between the supply chains, so that all companies are trying to integrate their supply chains. However, to really integrate well their suppliers form a highly efficient supply chain body is very difficult. To obtain a favorable position in the competition, but also to provide consumers with products meet the mind, we need an efficient supply chain. Companies need to enhance the competitiveness of the value chain must be integrated supply chain collaboration is the integration of decomposition and power value chain. For the enterprise value chain, the integration cannot ignore the aspect of the opponent's advantage as their own development, but also planning and cooperation between enterprises can also discover new opportunities, that is, to increase their competitive advantage. When preclude the use of supply chain collaborative way to complete value chain integration, although results of integration has some risks, but they have the flexibility and low cost properties is more appropriate choice.

\section{Related Works}

\subsection{Status of Logistics Scheduling}

The main problem is the logistics and distribution vehicle scheduling problem, since the introduction of its vehicle scheduling problem has been a hot research scholars around the problem modeling and solving algorithm published a large number of relevant literature, research in this part of predecessors literature briefly reviewed, introduced from 
the optimization target, modeling and algorithm in three aspects.

First, the optimum choices of destination; vehicle scheduling problem is that under certain constraints, choose a target as the optimization goal to achieve total distribution costs (broad distribution costs, such as delivery time, total cost, need vehicles, etc.) Optimal, wherein the total distribution costs are generally expressed as the following in some form or several forms of synthesis: 1) minimizing the total cost of the vehicle to complete the task of distribution of the costs and minimal, such as: vehicle enabled fixed costs, vehicle costs, vehicle wait costs, vehicle service costs, vehicle costs punishment. Wherein the vehicle is started showing the basic cost of fixed costs enabled vehicle, vehicle rental fees; vehicle running costs represent the cost of vehicles produced in the process of moving, such as fuel expenses; vehicle waiting costs represent costs incurred when the vehicle early to wait for service, the parking fees; vehicle service charges represent costs incurred when the vehicle service users, such as transport charges, etc.; punishment vehicle cost indicate vehicle delay time caused by fine service. [1] vehicle scheduling problem with time windows have been studied to optimize the objective is to reduce the gross vehicle with minimum cost; the literature [2] on the open vehicle routing problem with time windows have been studied to optimize the target vehicle to enable fixed fees and expenses and minimum vehicle; [3] in the transport vehicle minimum sum of direct costs and the shortest distance and the grid optimization target; [4] rental and variable costs to the minimum and, where available this is the unit becomes kilometers fuel consumption, improved cost vague concept to other literature, but there is a flaw, without considering the load of the vehicle is not fuel consumption while the unit is not the same, especially for large vehicle fuel consumption unit change is relatively large; [5] respectively, the distance to fuel costs and cost optimization objectives were analyzed, in order to obtain better fuel cost optimization goal to optimize the distribution network of the conclusions, but it does not take into account different models the use of different fixed costs; [6] Although the cost of the vehicle will be used for the breakdown of fixed and variable costs, but did not affect the variable costs important factor in vehicle rate analysis. 2) The minimum number of vehicles required to complete all distribution tasks required minimum total number of vehicles, assuming different distribution paths corresponding to the different distribution vehicle, the number of vehicles required minimum distribution is equivalent to the minimum number of paths. [7] cargo container terminal handling problems were studied, set up a buffer for temporary storage of cargo container terminals and warehouses were in the vehicle to dock before the buffer is full the goods to the warehouse of the buffer, its optimization to meet the target time window and dock buffer volume constraints under the premise of the total number of vehicles using the smallest; [8] use a two-stage local search technique for solving the vehicle routing problem with time windows, the first phase will be assigned to each user the total number of vehicles and vehicle smallest, the second stage in the path of the user to adjust the order so that the total minimum cost; [9] designed a four-stage algorithm for vehicle scheduling problem, the second stage of the algorithm to improve the initial solution, so that the required minimum number of vehicles; [10] proposed a hybrid heuristic algorithm to minimize the number of vehicles required for the target vehicle scheduling problem. 3 ) the minimum total distance, complete all distribution tasks Vehicle Routing minimum total length. [11] of the vehicle scheduling problem to minimize the total distance traveled, studied, and were using genetic algorithm, the problem is solved heuristic column generation heuristic algorithm and the like; [12] ant colony algorithm up to minimize the total distance traveled for the optimization target multi-distribution center vehicle scheduling problem; 4) the minimum service time to complete all distribution tasks total delivery time is minimized, where delivery time is generally includes a vehicle travel time, waiting time, service time and delays etc., in which the vehicle is traveling in time represents the time a vehicle on the path, waiting time represents the time waiting for service vehicles premature arrival. Secondly, modeling studies, modeling quantitative 
research vehicle scheduling problem of logistics distribution cost approach, the current mathematical model for vehicle routing problem of representation can be broadly grouped into three categories: vehicle flow model, material flow model and a set covering model. Material flow model material flow model is developed in recent years a class of vehicle routing problem model, which arc assigned for each variable used to represent the amount of cargo through the arc. Suppose that Yuan said vehicle through an arc $<i, j>$ the amount of supplies to the user, we can use material flow model to describe the distribution network of all the materials total logistics and distribution route, it can easily be extended to the energy sector, the use of material flow model describes the energy flow condition of the distribution network. Set covering models from graph theory point of view, the distribution network of the vehicle routing problem is actually a loop through the collection of all users feasible path and, therefore, from the point of view of set theory to describe the mathematical model, if all of the distribution network feasible path as a set, the optimum solution to the problem is to produce a divided minimal distribution costs of collection. Set covering model a feasible path allocation for each binary variable indicating whether the path to the optimal path, using a collection of processing methods to solve the problem.

\subsection{Supply Chain Status}

In the service supply chain, each node enterprise supply chain can not be like that, ready products before customers arrive by adjusting inventory to manage the supply chain, the supply chain can be managed by adjusting service capacity. In addition, the service supply chain is essentially based on the capability of cooperation based supply chain, due to the ability itself is intangible, difficult to measure and control, therefore, functional logistics service provider is likely in the cooperation process can not meet customers' changing needs capacity and forced-out, it is possible to complete the evaluation of the performance of the service can not meet the standard contract is terminated cooperation. Moreover, the logistics service supply chain is essentially based on the capability of cooperation based service supply chain. Logistics Service Supply Chain Integrators the background is in low self-service capabilities, logistics, need to outsource appropriate services to complete the operation; In addition, the ability to work, there are two cases, one service provider to integrate their lack of ability, and the second, integrators simply can not have this capability. Current inventory coordination is one of the main coordinating supply chain, due to the ability to inventory and supply chain service supply chain with similar properties, so the ability to work coordinated logistics service supply chain service supply chain coordination is an important part.

Functional logistics business logistics services provided by a single, standard, business development is generally restricted to a particular area, also called the single logistics company, it can only undertake or complete one or several logistics services, according to its engaged the main function can be divided into transport logistics enterprises, enterprise storage, distribution and processing enterprises, in building a network of national and even global, integrated logistics service providers tend to absorb them as suppliers. Integrated logistics service providers is a comprehensive logistics enterprises, its large-scale, well-funded, and there is a good business reputation, and even be able to take a number of all logistics services. But both belong to logistics companies, logistics companies (at least engaged in the transportation (including freight forwarders, express cargo) or a business warehousing business, and in accordance with customer demand for logistics and transport, storage, handling, packaging, distribution processing, distribution, etc. the basic functions of the organization and management, and information management systems have their own business with the city to adapt to, the implementation of independent accounting, independent bear civil liability of economic organization, unincorporated economic organization may apply mutatis mutandis logistics service supply chain is the core business of logistics services logistics service chain (such 
as logistics and procurement, transportation, warehousing, packaging, processing and distribution, etc.) and logistics end-users together into a whole functional network chain structure, joined the enterprise network chain logistics services in coordinating operations and compete to win in the process, share information, risks, joint decision-making, mutual benefit and common development. still from the perspective of integration of resources to improve the performance of the service supply chain, but each node is given a logical relationship between enterprises, research services for the latter specific issues chains provides structural model.

Logistics is a service, so has the characteristics of logistics capabilities service capabilities. Foreign global logistics research team by the largest research project logistics capabilities, with the kinds of methods to identify the species of common logistics capacity, and is divided into four categories: positioning, integration, agility and methods of measurement. Different companies to develop their core logistics capabilities based on market conditions and their own. The logistics capability is defined as an enterprise can at the lowest possible total cost of providing a competitive advantage relative evaluation of customer service. Referring to the situation, that the logistics capabilities into the overall management capabilities and the ability to add value in two parts, and demonstrate the impact on the overall logistics performance after leading both capabilities and cost and differentiation strategy in combination, namely logistics capabilities and business strategies are closely related, exists between business strategy and overall performance of the forward off. Ma Shihua (think logistics capability refers to a particular logistics system, customer demand from the receiving, processing orders, sorting goods, transportation to the delivery to the customer throughout the course of the operation, in response speed, logistics cost, time and order fulfillment delivery reliability Order comprehensive reflection of the other aspects. it includes the ability to transport goods (tangible factors) and organizational and management skills (intangible elements) to perform the logistics process. Liu Weihua, etc. (defined logistics capability from an operational point of view, the logistics services business in certain technical conditions and service level requirements, the highest value of output per unit time service enterprise has the logistics capability of constituting include hard and soft capability ability, capability refers to hard physical resources of the business, soft skills is an enterprise management level. Jiang Jifeng etc. (think logistics service capability is divided into two basic services and value-added service capabilities ability basic services refers to the transportation, storage, distribution and information services; value-added services refer to distribution processing, service convenience, customization and extensibility services.

\subsection{Research Status of Cloud Computing}

That communication services, corporate communications to the supplier, the supplier is responsible for operation and maintenance of their related hardware and software, the hardware and software are voice services, instant messaging, video conferencing will be the. Users can choose to deploy the entire corporate communications system, and to use to pay for services. Service providers are often packaged together, including integrated access traditional voice and data, advanced unified communications services as well as advanced user-level switch functions. It includes redundant switching, processing circuitry required for the diversity of its client, the client device redundancy and WAN failover solutions. With the flexibility and scalability features for small and medium enterprises to provide coverage using more capacity, equipment, service mode or regions according to their customers' needs, it can be set dynamically scale capacity of the equipment to keep up with customer demand and therefore service providers can achieve resource not to be wasted. Almost no supervision of the customer, thus eliminating the need for capital investment enterprise customers, reducing the cost of equipment operation and management of routine maintenance and infrastructure. What advantages 
does it have? First, as we mentioned operating expenses, it is the cost of the entire customer base to pay suppliers together, which is shared with this network than to establish an independent enterprise itself more economical. Second, because unified communications is outsourced to a service provider, the customer pays only the cost of the service, need to buy equipment, it will save money here. Again, select flexible and no additional expenditures technology obsolescence risk and technology upgrades, service providers do not have enough flexibility to be bound by a single vendor to invest in, they can use such as,,, Cisco, Microsoft, Nortel Networks, and so the best suppliers, which is more economical than select a single vendor, and companies are having to worry about technology obsolescence, outsourcing to a service provider in the future, they will absorb the burden on the user, constantly updated equipment. Finally, and most important thing is to ensure uninterrupted data, when the company suffered a sudden interruption of communication through the data center geographically dispersed can diversify risk, a multi-level redundancy in the system, reducing the risk. Infrastructure as a Service is the computer infrastructure as a service provided to the user. As long as connected to the network, we can buy storage, servers, etc. resources without having to purchase related equipment, customer use of the resources to pay for how much it costs, just like water and electricity to pay the same. One well-known providers the Amazon Elastic Compute Cloud and Mosso cloud server. Unlike previous lease a physical machine, server resource users acquire only after the real server virtualization resources, so make fuller use of the server, the server provider as capital investment will get more in return. Amazon allows users to increase or decrease based on the application requirements, dynamic scalability. Amazon (Elastic Block Storage) to provide persistent storage as an example, can be attached to any instance running as a standard block device, and automatically replicated back-end, create a data volume snapshot point in time, as the volume of the starting point for the Amazon, can indefinite protection of data. Amazon also provides one or more instances in multiple locations ability, and provide other available area in the same area inexpensive, low latency network connections. Clusters, grid computing, utility computing is very similar to on-demand computing, building blocks can achieve cloud computing model at this stage. Platform as a service-based applications run, it provides users with a software development platform, developers care based only on development and general care what operating system. Service allows developers to develop innovative energy are used, rather than complex hardware or software. Conventional software development needs require specific hardware, operating systems, databases and so on according to specific needs, the hardware and software environment to build a good future, developers can develop their applications in complex development platform, at the same time also need to network management, systems management and with the database management team, but also it needs a lot of power for a variety of servers to keep the system temperature. It provides developers with a faster and more cost-effective mode and needed to deploy applications on the network hardware, software, and other environments. PaaS feature is included in the development, deployment, testing, hosting and application management services, based on common standards and the like, or other special techniques. Suppliers typically include concurrency management, scalability, failover and security services. Another feature is the integrated database and on the platform, supports Simple Object Access Protocol and other interfaces, each distributed teams can share code, greatly improving the productivity of the product. Since the software as a service are written in code on the platform, so it is available to purchasers of software and services on the platform. Manufacturers will develop software deployed on their own servers, users according to their usage by subscription application to the provider, and access to these applications through the network, the unit is the amount of time to pay and service software. But users are not buying software vendors on rent server software, unlike using conventional software, users typically a one-time expenditure of considerable expense before it can use, but pay on demand, and is not responsible for 
software deployment, upgrades, maintenance, these tasks both to providers, they are responsible for.

\section{Proposed Scheme}

This paper presents a method based on a complex system of logistics scheduling random graph theory, the design of logistics vehicles, aircraft and rail and water transportation resources co-ordination arrangements, logistics distribution center logistics network as a node point, according to the complexity of the system according to the probability of a random graph Construction of the network, which can build a more reasonable logistics distribution and scheduling program, and this program is based on the mobile Internet, cloud computing environments, so the calculation speed and the mobilization of resources flow can be performed at a larger geographical environment, it has global optimization possible. According to a random graph scheduling can be applied stochastic programming, stochastic programming model commonly used in three ways: 1) expectations model, mathematical expectation to describe random variables, and further the stochastic programming problem into an ordinary linear programming; 2) chance constrained programming model, by reliability random variable random questions random objective function and constraints into the general type of objective function and constraints; 3) opportunities related programming model, probabilistic decision plan a great correlation random events.

\subsection{Stochastic Programming Logistics Scheduling}

Because of the random planning constraints $g_{j}(x, \xi) \leq 0$ often appear absolutely can not meet the needs of the problem by transforming the stochastic programming model, the chance constrained programming model is problematic for random variables in the constraint conditions, constraints and reliability through the objective function (constraint and the establishment of the objective function probability) to simplify decision-making program, the following is a chance constrained programming model:

$\min f(x, \xi)$

s.t. $\operatorname{Pr}\left(g_{j}(x, \xi) \leq 0\right) \geq \beta_{j}, j=1,2, \cdots, \mathrm{m}$

Wherein $\beta_{j} \in[0,1]$, as a function of the reliability constraint (ie, the probability function is not less than the establishment of the constraint ${ }^{\beta_{j}}$ )

A chance constrained programming model (1) is based on the standardized random variables,

$\xi^{*}=\frac{\xi-E(\xi)}{\sqrt{D(\xi)}}$

The mathematical expectation $E(\xi)$ of $\xi$ the variance $D(\xi)$, and $D(\xi) \neq 0$, $E\left(\xi^{*}\right)=0 D\left(\xi^{*}\right)=1$. Model (1) of the objective function $f(x, \xi)$ and constraint functions $g_{j}(x, \xi)$, their mathematical expectation and variance of the implementation of standardized, $\Psi_{a}$ standardized sub-sites (reliability coefficient) parameter $f(x, \xi)$, the standardization sub-sites $\Phi_{\beta_{j}}$ (reliability coefficient) parameter $g_{j}(x, \xi)$ in specific practical problems the combination of objective function and constraint distribution functions, decision-makers according to their own satisfaction, choose a reliable factor to give the following models: 


$$
\begin{aligned}
& \min \left[E(f(x, \xi))+\Psi_{a} \sqrt{D(f(x, \xi))}\right] \\
& \text { s.t. } E\left(g_{j}(x, \xi)\right)+\Phi_{\beta_{j}} \sqrt{D\left(g_{j}(x, \xi)\right)} \leq 0
\end{aligned}
$$

Since the introduction of reliability coefficient is the model (2) in the form of features, it may be called a stochastic programming model based on reliability coefficient. When the probability distribution of random variables is not clear, probably $f(x, \xi)$ in conjunction with the distribution ${ }^{g_{j}}(x, \xi)$ according to the following steps to select a reliable factor:

Step1 combination of $f(x, \xi) g_{j}(x, \xi)$ market conditions and policy makers will to choose and reliability;

Step2 by the method of theoretical analysis and statistical analysis, $f(x, \xi) g_{j}(x, \xi)$ to determine the characteristics and probably distributed;

Step3 $f(x, \xi) g_{j}(x, \xi)$ reliability and according to the distribution characteristics and selected by means of calculation to determine the phase look-up table $\Psi_{a}$ and $\Phi_{\beta_{j}}$ the reliability coefficient .

Step4 reliability coefficient $\Psi_{a \text { and }} \Phi_{\beta_{j}}$ different values by the model (2) to obtain the optimal solution will be different, choose a reliable factor to reflect the effectiveness of the model of decision-making, but also for solving stochastic programming model is the key.

Describes the actual problem in the form of abstract linear programming, reflects the essence of the law among the number of things objectively, taking into account all factors that may affect the logistics and distribution, to seize the nature of the problem, the model simply determined expectation and variance of random variables can be, based on market conditions in accordance with the wishes of policy makers to select a reliability factor, without calculating the probability of a random variable, with the Lingo12.0 software makes operation easy operation. Most real-life problems contain uncertainties have wide stochastic model (3) the scope of application, practical issues such as production organization and planning, assignment problem, layout issues, problems, and so reasonable ingredients. With model (3) can be solved in accordance with the following steps of:

Step 1 Calculate shipping cost and supply and demand factors of expectation and variance;

Step 2 find $z=\sum_{i=1}^{m} \sum_{j=1}^{n} c_{i j} x_{i j}$ the mean and variance;

Step 3 decision maker satisfaction according to their willingness to choose the appropriate reliability coefficient (coefficient to choose a reliable supply and demand changes will affect the amount and distribution activities), listed in numerical linear programming equation;

Step 4 Open Lingo12.0 software programming solver, draw the optimal distribution program.

Random search traffic scheduling genetic algorithm, the steps of

The first step, for the vehicle scheduling problem of $n$ customers, uses a $2 n \llbracket n$ three dimensional quantum bit matrix to represent a quantum chromosome, so that the construction of the bit matrix chromosome, constitutes the initial population $Q(t)$. Decoding transform adopts the two-step method of "group after the first line", the first step is to generate a sequence of customer service, by generating random numbers in $[0,1]$ 
to generate the $m \llbracket m$ two-dimensional 0-1 observation matrix, search and adjust the matrix to ensure that each row and column of the matrix is only a 1, the abscissa represents the order of service, the ordinate represents the customer number, for example, the problem of 5 customers, $0-1$ matrix is as follows:

$$
\left[\begin{array}{llll}
1 & 0 & 0 & 0 \\
0 & 0 & 1 & 0 \\
0 & 1 & 0 & 0 \\
0 & 0 & 0 & 0
\end{array}\right]
$$

The second step is to form the vehicle driving route, enabling a car at a time, according to the customers' order sequence table service customers, when the current vehicle can not meet the needs of the next customer, to enable a car, so you can convert the bit coding to integer coding, such as $(0,1,3,2,0,5,4,0)$, represents five customers demand schedule need two cars to finish, 0 represents the distribution center. If the required number of vehicles exceeds the number of vehicles, this path is not feasible, need to regenerate a new quantum bit chromosome, so that the bits population $\mathrm{Q}(\mathrm{t})$ can be converted to an integer population $\mathrm{P}(\mathrm{t})$.

The transfer of states in quantum evolutionary algorithm is realized by the quantum gate transformation matrix, and the individual evolution is guided by quantum gates, its structure is the key to design quantum evolutionary algorithm, directly affect the performance of the algorithm. According to the Schrodinger equation, the quantum gate should meet $U^{\prime}=1$. The design of quantum gates in a variety of ways, such as NAND gates, controlled NOT gate, Hadamard transform gate, the most common quantum rotating gate as shown in (5):

$\left[\begin{array}{c}\alpha_{(m, n)}^{t+1} \\ \beta_{(m, n)}^{t+1}\end{array}\right]=U(\delta \theta)\left[\begin{array}{c}\alpha_{(m, n)}^{\prime} \\ \beta_{(m, n)}^{\prime}\end{array}\right]$

The third step, the calculation of sufficiency.

Each chromosome in the population after decoding transform, the chromosome is changed into the integer, calculate its objective function value $\mathrm{Z}$; if the chromosome violates the constraint condition, give $\mathrm{Z}$ a very big number. In this paper, the sufficiency function of the chromosomes is consistent with the objective function, thus, the smaller the function value, the better the chromosome.

The fourth step, the cross operation of the route chromosomal.

\subsection{Scheduling Implementation based on Cloud Computing}

(1) Vertical handover decision algorithm based on mobile communication system, mobile computing environment handover decision algorithm is to determine whether the current environment to achieve the switching condition occurs, consider switching to achieve the switching condition that is otherwise execution continues in the original network. Since there is no set minimum performance standards, as long as each test to find better execution loop or the current network is no longer available, switch ensue. This means that every time the detection of network parameters is available nearby, the criteria for determining whether to trigger the switch to switch, network selection algorithm decided handover target network. Network selection algorithm is an attempt to find a network, the network does not attribute parameter corresponding to the difference parameters than the current network, and at least one parameter is a significant improvement. To achieve the above comparison, it should meet in two consecutive test, with a load on the network does not change, and the corresponding parameters are fixed values. Accordingly, the scope of the characterizing parameter values used in the algorithm is "performance equal" or "performance has been significantly improved." Herein considered a "performance equal" attribute parameter value is better than the original attribute parameter value times an 
arbitrary value greater than (e.g., fetch, and is considered the property parameter value "improved properties" is better than the original attribute parameter value fold or better performance, greater than any value. the purpose of this work is not found over the value of these thresholds (dynamic or general), but assumes some reasonable threshold to test their usefulness the handover decision criterion in this paper are: guidelines current network is no longer available, the current network handoff criteria is available, but there are new alternative network and to meet the conditions, the switching conditions of all the candidates attribute parameter must be greater than equal to the current attribute parameter corresponding to the network is equivalent to the minimum multiplying factor. All attribute parameter conditions of the candidates at least one parameter of greater than or equal to the corresponding properties of the current network parameter times the minimum improvement factor.

(2) In the guidelines, not only trying to decide whether to switch improved, taking into account issues related to the degree of improvement. Of course, all the attributes of the parameters a candidate network will meet the minimum improvement factor requirements, the network is an ideal candidate network; a part attribute parameter candidate network to meet the minimum improvement factor requirements of the network is relatively good candidate network; a no improvement factor attribute parameters to meet the minimum requirements for a candidate network, the network is not a candidate network handover. In the experiment, the paper used in an appropriate threshold factor as a minimum to trigger switching. This threshold value can be verified through the migration strategy to correct.

\section{Mobile Cloud Platform}

There is a huge difference between the vehicle scheduling system based on cloud distribution mode and the traditional vehicle scheduling system. The traditional vehicle scheduling system needs manual input distribution tasks, and the task is relatively simple, so we do not need to establish a relatively large database. The vehicle scheduling system based on cloud distribution mode is a distribution task which is matched by the cloud service distribution platform. The system can optimize the route according to the matching distribution task, and then the optimization scheme is generated, so the structure and function of the system is relatively high. Its implementation topology shown in Figure 1

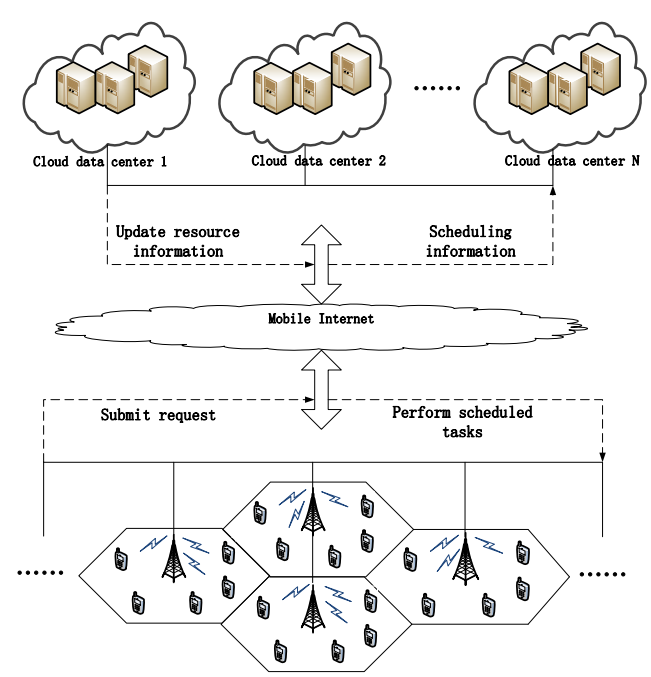

Figure 1. Mobile Internet Cloud Computing Model 


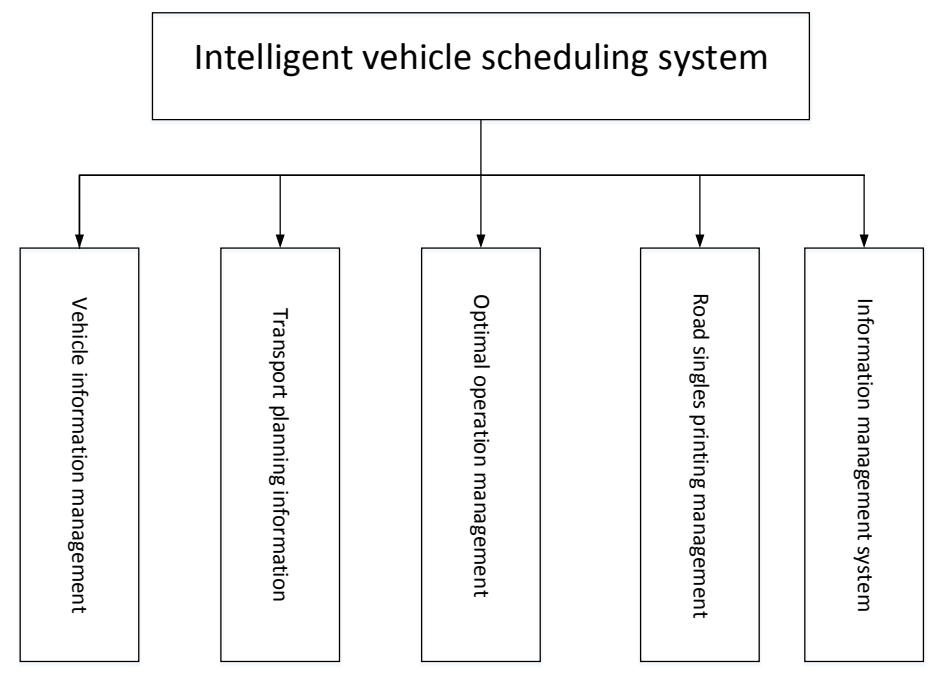

Figure 2. System Model

The optimal scheduling management module is the core of the intelligent vehicle scheduling system and other modules around it. Firstly, according to the matching distribution task, the system carries on the analysis of distribution vehicle condition and analyze whether need additional vehicles in the distribution process. When the vehicle is determined, intelligent algorithm in the optimization scheduling module starts to optimize the production scheme, and convert it to distribution waybill.

Specific operating structure is as follows.

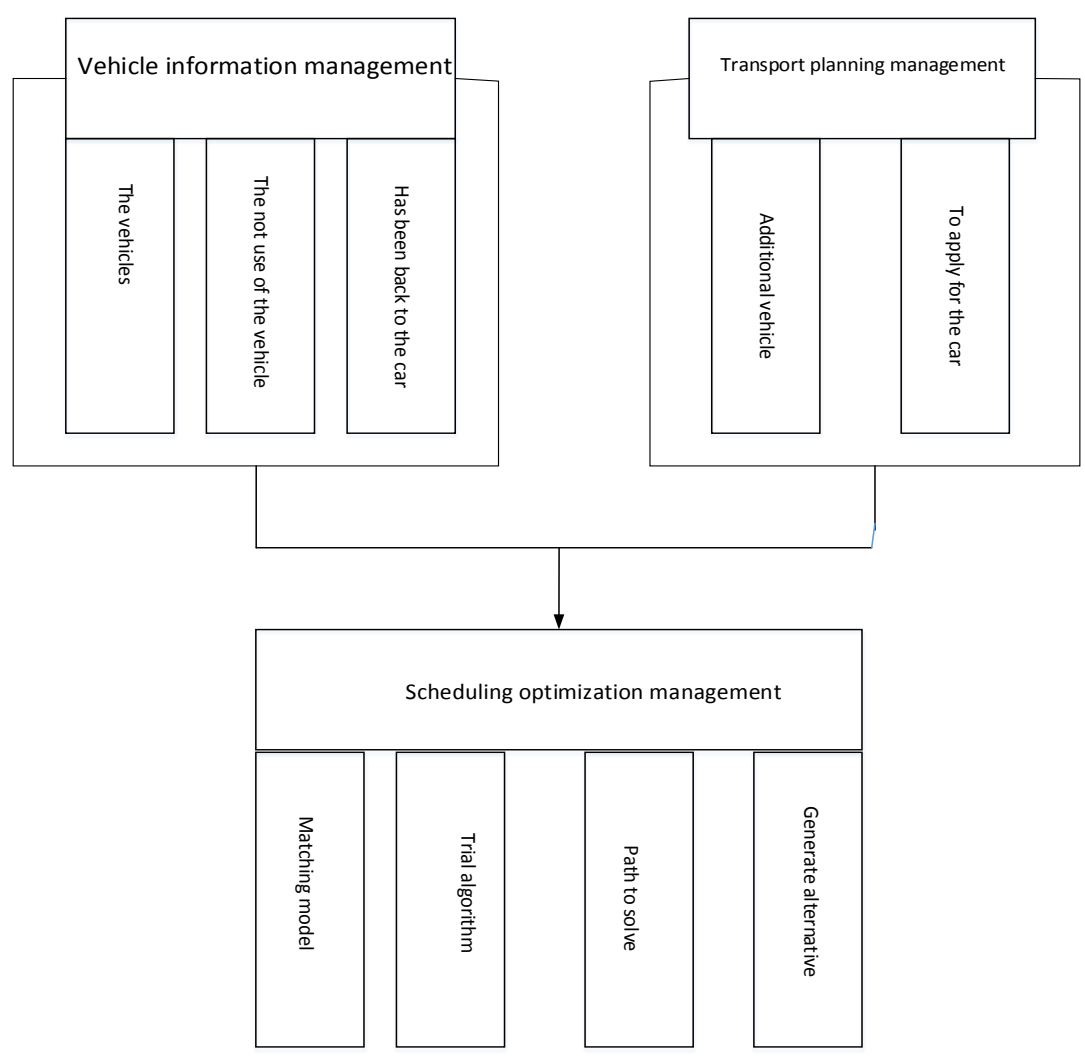

Figure 3. The Scheduling System 


\section{Conclusion}

Based on the principle of stochastic programming complex system of random graphs, scheduling information and scheduling logistics transportation load tool optimized for this program by means of the network structure of the mobile Internet cloud computing, scheduling can be more comprehensive in the larger region program, having the most global sexual characteristics. Subsequent analysis of big data, to be follow-up continued to deepen.

\section{Acknowledgement}

This work was all supported by the National Natural Science Foundation of China under Grant 613600124 , the Soft Science Project of HeNan Science and Technology Department under grant No.132400411365 and Key scientific research projects of Henan Province Colleges and Universities under grant NO. 15B520046.

\section{References}

[1] Khorshed M T, Ali A B M S, Wasimi S A. A survey on gaps, threat remediation challenges and some thoughts for proactive attack detection in cloud computing[J]. Future Generation computer systems, 2012, 28(6): 833-851.

[2] Chandrasekaran M, Muralidhar M, Dixit U S. Online optimization of multipass machining based on cloud computing[J]. The International Journal of Advanced Manufacturing Technology, 2013, 65(1-4): 239-250.

[3] Stantchev V, Prieto-González L, Tamm G. Cloud computing service for knowledge assessment and studies recommendation in crowdsourcing and collaborative learning environments based on social network analysis[J]. Computers in Human Behavior, 2015.

[4] Lu C W, Hsieh C M, Chang C H, et al. An improvement to data service in cloud computing with content sensitive transaction analysis and adaptation[C]//Computer Software and Applications Conference Workshops (COMPSACW), 2013 IEEE 37th Annual. IEEE, 2013: 463-468.

[5] Hosseininaveh A, Sargeant B, Erfani T, et al. Towards fully automatic reliable 3D acquisition: From designing imaging network to a complete and accurate point cloud[J]. Robotics and Autonomous Systems, 2014, 62(8): 1197-1207.

[6] Eberbach E, Mikkilineni R. Cloud Computing with DNA Cognitive Architecture in the Context of Turing's" Unsinkable" Titanic Machine[C]//WETICE Conference (WETICE), 2014 IEEE 23rd International. IEEE, 2014: 125-130.

[7] Cheng C C, Cheng F C, Lin P H, et al. A Cloud-Computing Local Histogram Construction Algorithm for Big Image Data[C]//Big Data (BigData Congress), 2014 IEEE International Congress on. IEEE, 2014: 200-203.

[8] Li H M, Lu J J, Wei C. Research of coal mine safety monitoring and early warning system based on cloud computing[J]. Gongkuang Zidonghua-Industry and Mine Automation, 2013, 39(3): 46-49.

[9] Chandrasekaran M, Muralidhar M, Dixit U S. Online optimization of a finish turning process: strategy and experimental validation[J]. The International Journal of Advanced Manufacturing Technology, 2014, 75(5-8): 783-791.

[10] Piscini A, Picchiani M, Chini M, et al. A neural network approach for the simultaneous retrieval of volcanic ash parameters and SO2 using MODIS data[J]. Atmos. Meas. Tech. Discuss., 2014, 7: 3349-3395.

[11] Chaudhry M T, Ling T C, Manzoor A. Considering thermal-aware proactive and reactive scheduling and cooling for green data-centers[C]//Advanced Computer Science Applications and Technologies (ACSAT), 2012 International Conference on. IEEE, 2012: 87-91.

[12] Han K H, Kim J H. Quant um2inspired evolutionary algorithm for a class of combinatorial optimization[J].IEEE Trans on Evolutionary Computation,2002,6(6):580-593.

[13] Hichem TALBI, Amer DRAA, Mohamed BATOUCHE. A New Quantum-Inspired Genetic Algorithm for Solving the Traveling Salesman Problem[C].IEEE International Conference on Industrial Technology,2004: 1192-1197. 